\title{
COMPARISON OF A SIMPLE AND A DETAILED MODEL OF MAGNETIC HYSTERESIS WITH MEASUREMENTS ON ELECTRICAL STEEL
}

\author{
Hanif Tavakoli ${ }^{1}$, Dierk Bormann ${ }^{2}$, David Ribbenfjärd ${ }^{1}$, Göran Engdahl ${ }^{1}$ \\ ${ }^{1}$ Royal Institute of Technology, School of Electrical Engineering, Teknikringen 33 SE-100 44 Stockholm, Sweden, \\ e-mail: hanif.tavakoli@ee.kth.se, david.ribbenfjard@ee.kth.se, goran.engdahl@ee.kth.se \\ ${ }^{2}$ ABB AB - Corporate Research, Power Technologies, Utvecklingsgränd 6, S-721 78 Västerås, Sweden, e-mail: \\ dierk.bormann@se.abb.com
}

\begin{abstract}
For efficient magnetic field calculations in electrical machines, the hysteresis and losses in laminated electrical steel must be modeled in a simple and reliable way. In this paper, a frequency dependent complex permeability model and a more detailed model (describing hysteresis, classical eddy current effects, and excess losses separately) are compared to singlesheet measurements. It is discussed under which circumstances the simple complex- $\mu$ model is an adequate substitute for the more detailed model.
\end{abstract}

\section{INTRODUCTION}

Recent research has resulted in detailed models of the magnetic hysteresis and loss mechanisms in a wide frequency range $[1,2,3]$. Although these models provide a good description of magnetic material properties or of simple reluctance circuits based on them, they are too demanding numerically to be incorporated into a full-scale magnetic field simulation of a realistic geometry, as with a FEM or FDM calculation tool. In other words, while such a detailed simulation of the $H-B$ relation of a single or a few interacting cells is still perfectly feasible, simulating thousands or ten thousands of them simultaneously may be inconvenient or impossible.

Moreover, in many practical situations a detailed description is not required either. Often the goal is to obtain a good estimate of some local or global quantity containing much less information than the detailed local $H-B$ relation, such as the local losses causing dangerous hot spots, or simply the total losses in a machine relevant for cooling or economic reasons. For such applications it is desirable to use a simple model of magnetic hysteresis and losses, which can easily be incorporated in field calculation tools but which at the same time is sufficiently close to reality, within the frequency range of interest for the specific application. Such a model is the description of magnetic (meta-)materials by a suitable frequency dependent complex permeability, which is the most general linear description of a local and isotropic $H-B$ relation. If desired, it can easily be extended to a nonlocal and/or anisotropic $H-B$ relation by turning $\mu$ from a scalar function of position into a distance dependent integral kernel and/or tensor, respectively [4].

In this paper, we discuss to which extent the measurement results obtained with a single-sheet tester on strips of electrical steel can reliably be described by a simple complex permeability function of frequency. Both the resulting $H-B$ curves and the effective complex permeability are compared to the measured data at different frequencies.
For comparison, we also report simulation results obtained with a much more detailed model of the magnetic hysteresis, eddy current and excess losses.

\section{THE COMPLEX-PERMEABILITY MODEL}

Reduced to its simplest terms, hysteresis introduces a time phase difference between $B$ and $H$. $B$ is assumed to lag $H$ by a constant angle $\theta_{\mathrm{h}}$ called the hysteresis angle. In such a description, harmonics introduced by saturation are ignored, and the hysteresis loop becomes an ellipse whose major axis is inclined by an angle $\theta_{\mathrm{h}}$ relative to the $H$-axis. Using complex field components $\hat{B}$ and $\hat{H}$, we can define a low-frequency complex permeability including hysteresis as

$$
\hat{\mu}_{\mathrm{h}}=\frac{\hat{B}}{\hat{H}}=\mu_{0} \mu_{\mathrm{r}} \mathrm{e}^{-\mathrm{j} \theta_{\mathrm{h}}} .
$$

In addition to this, eddy currents in the lamination sheets introduce frequency dependence. We briefly sketch the wellknown procedure [5] for deriving the effective frequency dependent complex permeability. Faraday's law

$$
\nabla \times \mathbf{E}=-\frac{\partial \mathbf{B}}{\partial t}
$$

and Ampere's law

$$
\nabla \times \mathbf{H}=\mathbf{J}+\frac{\partial \mathbf{D}}{\partial t},
$$

in combination with the constitutive relations

$$
\mathbf{J}=\sigma \mathbf{E}, \mathbf{D}=\varepsilon \mathbf{E}, \mathbf{B}=\hat{\mu}_{\mathrm{h}} \mathbf{H},
$$

and time-harmonic assumption lead to

$$
\nabla^{2} \mathbf{H}=\hat{\alpha}^{2} \mathbf{H}=\mathrm{j} \omega \hat{\mu}_{\mathrm{h}}(\sigma+\mathrm{j} \omega \varepsilon) \mathbf{H} .
$$

For lower frequencies when wave propagation can be ignored (i.e., $\sigma>\omega \varepsilon$ ), we have $\hat{\alpha}^{2} \approx \mathrm{j} \omega \hat{\mu}_{\mathrm{h}} \sigma$.

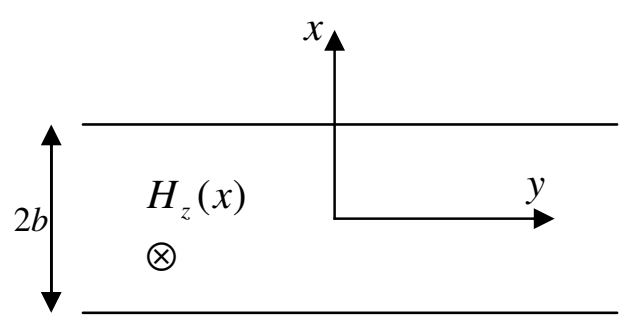

Fig. 1. Laminate infinite in $z$ direction, with a width in $y$ direction much larger than its thickness $2 b$, exposed to a $H$ field in $z$ direction.

For analysis of the magnetic field in a laminate, the simple geometry illustrated in Fig. 1 is appropriate. The magnetic 
field is applied in the $z$ direction, hence the only component of the magnetic field strength is $H_{z}$ which varies only in the $x$ direction, $H_{z}=H_{z}(x)$. In one dimension, eq. (5) reduces to

$$
\frac{\partial^{2} H_{z}}{\partial x^{2}}=\hat{\alpha}^{2} H_{z},
$$

which has the general solution

$$
H_{z}(x)=A_{1} \mathrm{e}^{\hat{\alpha} x}+A_{2} \mathrm{e}^{-\hat{\alpha} x} .
$$

The field strength on the both sides of the laminate is assumed to be $H_{0}$. For the reason of symmetry the following condition is obtained

$$
H_{z}(b)=H_{z}(-b)=H_{0} .
$$

The final expression for the magnetic field strength then becomes

$$
H_{z}(x)=H_{0} \frac{\cosh (\hat{\alpha} x)}{\cosh (\hat{\alpha} b)} .
$$

The effective, complex permeability of a lamination is given as the average magnetic flux density $\bar{B}$ in the laminate normalized to the surface magnetic field strength $H_{0}$,

$$
\begin{aligned}
\hat{\mu}_{\text {eff }} & =\mu_{\text {eff }}^{\prime}-\mathrm{j} \mu_{\text {eff }}^{\prime \prime} \\
& =\frac{\bar{B}}{H_{0}}=\frac{1}{H_{0} 2 b} \int_{-b}^{b} \hat{\mu}_{\mathrm{h}} H_{z}(x) \mathrm{d} x=\hat{\mu}_{\mathrm{h}} \frac{\tanh (\hat{\alpha} b)}{\hat{\alpha} b} .
\end{aligned}
$$

This expression accounts for the effect of hysteresis without saturation, and the effect of eddy currents. We assume here that additional (or "excess") losses are either negligible or have a similar frequency dependence so that they can be incorporated in the expression (10) for $\hat{\mu}_{\text {eff }}$.

\section{THE DETAILED HYSTERESIS MODEL}

Later in this paper we will report some results obtained with a more detailed model of the magnetic hysteresis, eddy current and excess losses, which is therefore described here in some detail.

The total hysteresis is a combination of three different phenomena, namely, static hysteresis, eddy current effects and excess eddy currents. For the detailed hysteresis model, the following approach has been used. The static hysteresis is modeled using Bergqvist's lag model [6, 7], the classical eddy currents are modeled using Cauer circuits $[9,3]$, and the excess losses are modeled using an approach by Bertotti [1].

\section{Static hysteresis}

The lag model of static hysteresis starts from the idea that the magnetic material consists of a finite number of pseudo particles $n_{p}$, i.e., volume fractions with different magnetization.

The total magnetization is then a weighted sum of the individual magnetization of all pseudo particles.
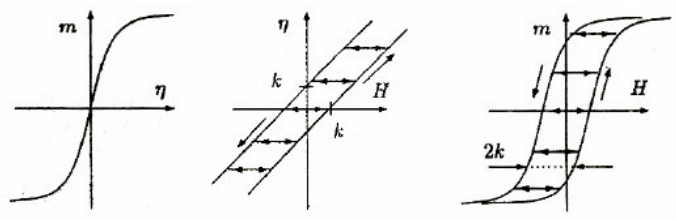

Fig. 2. Anhysteretic curve (left), play operator (middle), and resulting hysteresis curve (right); figure taken from [2].
The hysteresis curve for one particle is introduced by applying a "play operator" with "pinning strength" $k$ (which will determine the width of the hysteresis curve) on the anhysteretic curve, see Fig. 2.

Using a population of pseudo particles with different pinning strengths allows to construct minor loops. We assign an individual pinning strength $\lambda_{i} k$ to every pseudo particle, where $k$ is the mean pinning strength, and the $\lambda_{i}$ are dimensionless numbers. The total magnetization is then given by a weighted superposition of the contributions from all pseudo particles (Fig. 3).

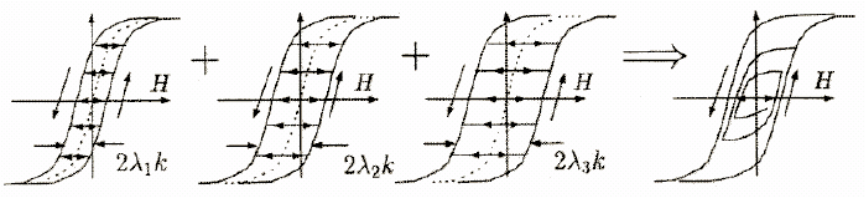

Fig. 3. Weighted superposition of the contributions from pseudo particles describes a minor loop; figure taken from [2].

The expression

$$
M_{\text {an }}(H)=\frac{2}{\pi} M_{\mathrm{s}} \arctan \left(\frac{\pi H \chi}{2 M_{\mathrm{s}}}\right)
$$

is used for the anhysteretic magnetization, where $M_{\mathrm{s}}$ is the magnetization saturation and $\chi$ is the susceptibility at $H=0$. The total magnetization of the material is then given by

$$
M=c M_{\text {an }}(H)+\int_{0}^{\infty} M_{\text {an }}\left(P_{\lambda k}(H)\right) \varsigma(\lambda) \mathrm{d} \lambda,
$$

where $c$ is a constant that governs the degree of reversibility, and the integral describes the hysteretic behavior (irreversible part). $P_{\lambda k}$ is a play-operator with the pinning strength $\lambda_{i} k$, and $\varsigma(\lambda)$ is a weight function describing the density of the pseudo particles. Finally, the magnetic flux density is obtained from $B=\mu_{0}(H+M)$.

Excess losses

Excess losses are caused by microscopic eddy currents induced by local changes in flux density due to domain wall movements. For our detailed model we use an approach described by Bertotti [1]. In this approach a number of active correlation regions are assumed randomly distributed in the material. The correlation regions are connected to the microstructure of the material like grain size, crystallographic textures and residual stresses. In Bertotti's model, the resulting contribution to the magnetic field strength is given by

$$
H_{\text {excess }}=\frac{n_{0} V_{0}}{2}\left(\sqrt{1+\frac{4 \sigma G 2 b w}{n_{0}^{2} V_{0}}\left|\frac{\mathrm{d} B}{\mathrm{~d} t}\right|}-1\right) \operatorname{sign}\left(\frac{\mathrm{d} B}{\mathrm{~d} t}\right),
$$

where $w$ is the width of the laminate and $2 b$, as before, its thickness. $G$ is a parameter depending on the structure of the magnetic domains. $n_{0}$ is a phenomenological parameter related to the number of active correlation regions when the frequency approaches zero, whereas $V_{0}$ determines to which extent micro-structural features affect the number of active correlation regions.

The parameters $n_{0}$ and $V_{0}$ are by definition frequency independent, but they are expected in reality to depend on the amplitude of the $B$ field [8]. Since the precise form of this 
dependence is unknown, their values are usually adjusted empirically for given amplitude. In the simulations reported here we use one set of (empirically determined) values, although the amplitude of the $B$ field slightly varies in the measurements.

\section{THE MEASUREMENT SETUP}

The magnetic measurements were carried out using a Single Sheet Tester. It consists of two equal U-shaped yokes placed face-to-face to each other (Fig. 4). The magnetic sheet to be tested is placed between the yokes and most of the flux is forced through it due to its high permeability. For the measurement of the flux in the test material a coil is surrounding the strip which is connected to a flux meter. The magnetic field strength is measured with a Hall probe placed close to the surface of the sample and connected to a Tesla meter. A sinusoidal $H$ field was applied to the sample; the $H$ and $B$ field values were measured for 100 periods and numerically filtered. Thereafter, the mean values at different phase angles of the $B$ and $H$ fields were calculated. These values were then used in the paper.

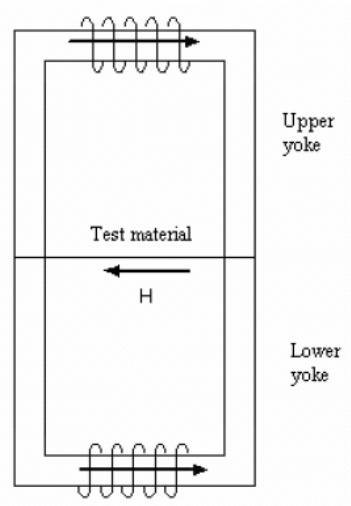

Fig. 4. Cross sectional view of our Single Sheet Tester.

We approximate the measured $H-B$ curve with a complex- $\mu$ ellipse characterized by the permeability $\hat{\mu}_{\text {meas }}$ by matching both its peak values $H_{\mathrm{p}}, B_{\mathrm{p}}$ and its area $A$ to the measured results. This is of course appropriate as long as the shape of the measured $H-B$ curve is close to an ellipse, i.e., if saturation effects are not too pronounced. The area $A$, which measures the power loss per cycle, is given by the integral

$$
A=\oint B_{\text {meas }} \mathrm{d} H_{\text {meas }}=\int_{0}^{T} B_{\text {meas }} \frac{\mathrm{d} H_{\text {meas }}}{\mathrm{d} t} \mathrm{~d} t,
$$

where $B_{\text {meas }}$ and $H_{\text {meas }}$ are the time dependent measured $B$ and $H$ fields, respectively, and $T$ is the duration of a period. If the measured magnetic field strength is assumed to vary sinusoidally,

$$
H_{\text {meas }}(t)=\operatorname{Re}\left(H_{\mathrm{p}} \mathrm{e}^{\mathrm{j} \omega t}\right)=H_{\mathrm{p}} \cos (\omega t),
$$

then its derivative becomes

$$
\frac{\mathrm{d} H_{\text {meas }}(t)}{\mathrm{d} t}=-\omega H_{\mathrm{p}} \sin (\omega t),
$$

and the measured magnetic flux density

$$
\begin{aligned}
B_{\text {meas }}(t) & =\operatorname{Re}\left(\hat{\mu}_{\text {meas }} H_{\mathrm{p}} \mathrm{e}^{\mathrm{j} \omega t}\right) \\
& =\operatorname{Re}\left(\left(\mu_{\text {meas }}^{\prime}-\mathrm{j} \mu_{\text {meas }}^{\prime \prime}\right) H_{\mathrm{p}} \mathrm{e}^{\mathrm{j} \omega t}\right) \\
& =H_{\mathrm{p}}\left(\mu_{\text {meas }}^{\prime} \cos (\omega t)+\mu_{\text {meas }}^{\prime \prime} \sin (\omega t)\right) .
\end{aligned}
$$

By inserting Eqs. (15) and (16) into (14) we get

$$
\mu_{\text {meas }}^{\prime \prime}=-\frac{A}{\pi H_{\mathrm{p}}^{2}} \text {. }
$$

Furthermore, from the relation $\left|\hat{\mu}_{\text {meas }}\right| H_{\mathrm{p}}=B_{\mathrm{p}}$ we obtain

$$
\left|\hat{\mu}_{\text {meas }}\right|^{2}=\left(\mu_{\text {meas }}^{\prime}\right)^{2}+\left(\mu_{\text {meas }}^{\prime \prime}\right)^{2}=\left(\frac{B_{\mathrm{p}}}{H_{\mathrm{p}}}\right)^{2},
$$

which implies

$$
\mu_{\text {meas }}^{\prime}=\sqrt{\left(\frac{B_{\mathrm{p}}}{H_{\mathrm{p}}}\right)^{2}-\left(\mu_{\text {meas }}^{\prime \prime}\right)^{2}} .
$$

Both $\mu_{\text {meas }}^{\prime}$ and $\mu_{\text {meas }}^{\prime \prime}$ are functions of frequency.

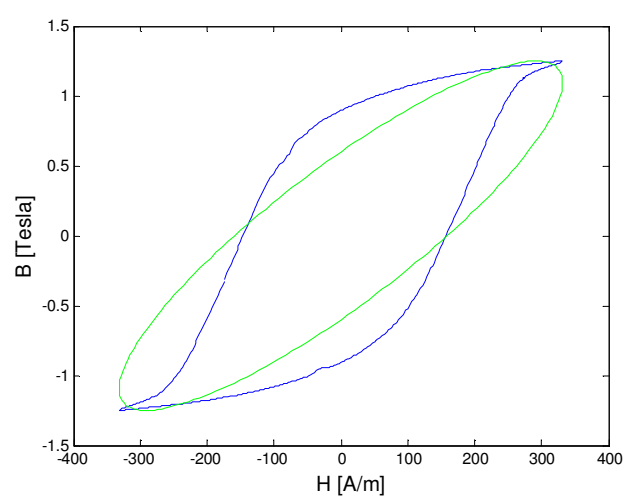

(a)

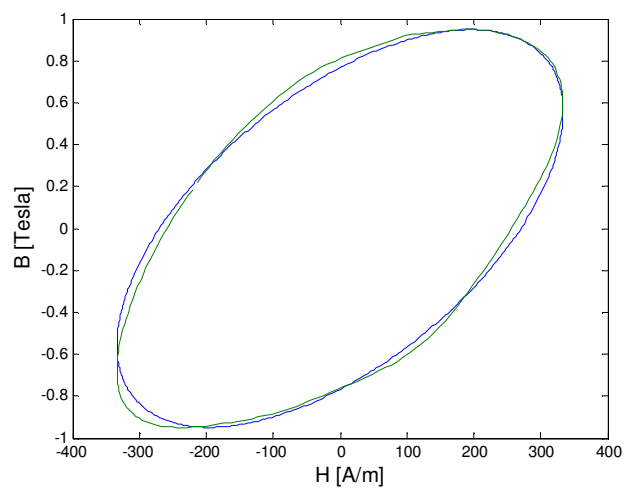

(b)

Fig. 5. $\quad H$ - $B$-curves from measurements (blue) and complex- $\mu$ model (green) with $\hat{\mu}_{\text {meas }}=\mu_{\text {meas }}^{\prime}-\mathrm{j} \mu_{\text {meas }}^{\prime \prime}$, for (a) $f=50 \mathrm{~Hz}$ and (b) $f=400 \mathrm{~Hz}$.

Figure 5 compares the measured $H-B$ curves with complex- $\mu$ ellipses, generated with the adapted $\hat{\mu}_{\text {meas }}$ at frequencies $f=$ $50 \mathrm{~Hz}$ and $400 \mathrm{~Hz}$.

$\hat{\mu}_{\text {eff }}$ as defined in (10) is a function of frequency and of a vector $\mathbf{x}=\left(\mu_{\mathrm{r}}, \theta_{\mathrm{h}}, \sigma b^{2}\right)$ containing the model parameters. It is adjusted to measured data by numerically minimizing the expression

$$
\sum_{i=1}^{N}\left|\hat{\mu}_{\text {eff }}\left(\mathbf{x}, \omega_{i}\right)-\hat{\mu}_{\text {meas }}\left(\omega_{i}\right)\right|^{2}
$$

with respect to $\mathbf{x} \cdot \hat{\mu}_{\text {meas }}\left(\omega_{i}\right)$ are the measured complex permeability values, defined by (18) and (20), at $N$ different frequencies $\omega_{i}=2 \pi f_{i}, i=1, \ldots, N$. We performed measurements at $N=9$ different frequencies ranging from $50 \mathrm{~Hz}$ to $2 \mathrm{kHz}$ (see Figs. 6 and 7 below), on a $100 \mathrm{~mm} \times 3.2 \mathrm{~mm}$ strip of the non-oriented magnetic material M600 with a thickness of $2 b=0.5 \mathrm{~mm}$. 


\section{RESULTS AND DISCUSSION}

Since the measurement setup was quite sensitive to noise, the measurements had to be numerically filtered. Adjusting $\hat{\mu}_{\text {eff }}$ to the filtered data using Eq. (21), we obtain the following model parameter values: $\mu_{\mathrm{r}}=3366, \theta_{\mathrm{h}}=0.477 \mathrm{rad}$, and $\sigma b^{2}=0.243 \mathrm{Sm}$, i.e., $\sigma=3.89 \times 10^{6} \mathrm{~S} / \mathrm{m}$. The last value is somewhat larger than the true dc conductivity of this material, $\sigma_{\mathrm{dc}}=3.33 \times 10^{6} \mathrm{~S} / \mathrm{m}$, since we have included here excess losses in the classical phenomenological form (10).

In Figure 6, the real and imaginary parts of the measured complex permeability are compared at different frequencies with the adjusted $\hat{\mu}_{\text {eff }}$.

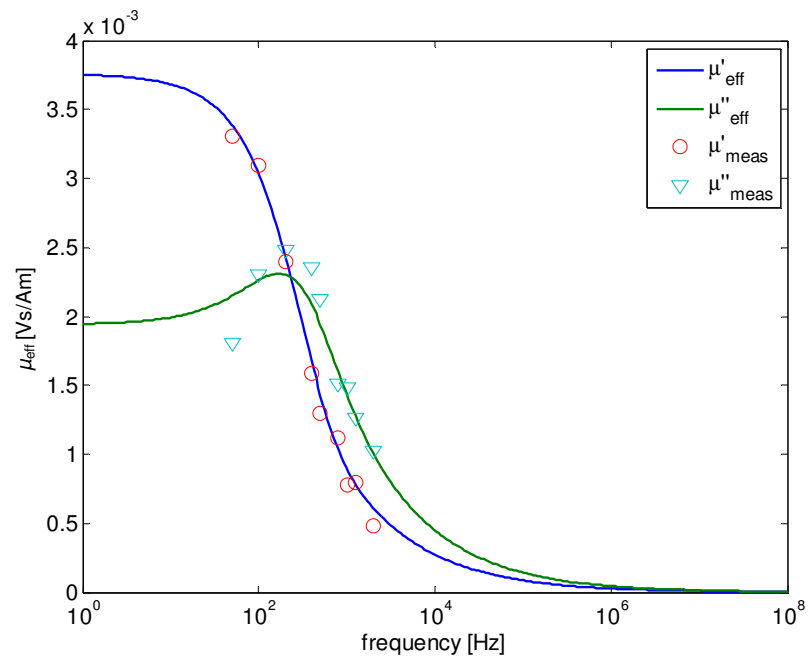

Fig. 6. Real and imaginary parts of the measured complex permeability (symbols) and of the fitted permeability function (curves).

The agreement is quite satisfactory considering the simplicity of the model, especially at higher frequencies. The deviation between $\mu_{\text {meas }}^{\prime \prime}$ and $\mu_{\text {eff }}^{\prime \prime}$ at the lowest frequencies is probably due to saturation effects which are not properly taken into account by the expression (10) for $\hat{\mu}_{\text {eff }}$, see for instance the measurement at $50 \mathrm{~Hz}$ (Fig. 5(a)). The amplitude had to be chosen large enough for the signal not to be covered by noise. Below the $H-B$ hysteresis curves are shown for all measured frequencies. Measurement, simple model, and detailed model are represented by solid green lines, dashed blue lines and dotted red lines, respectively.

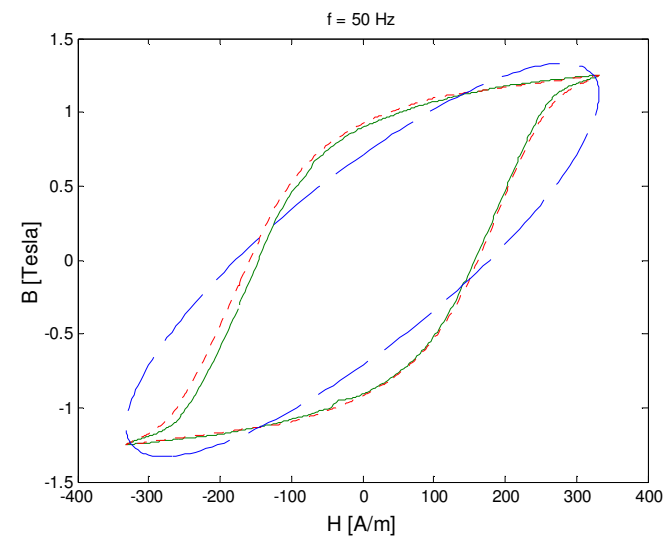

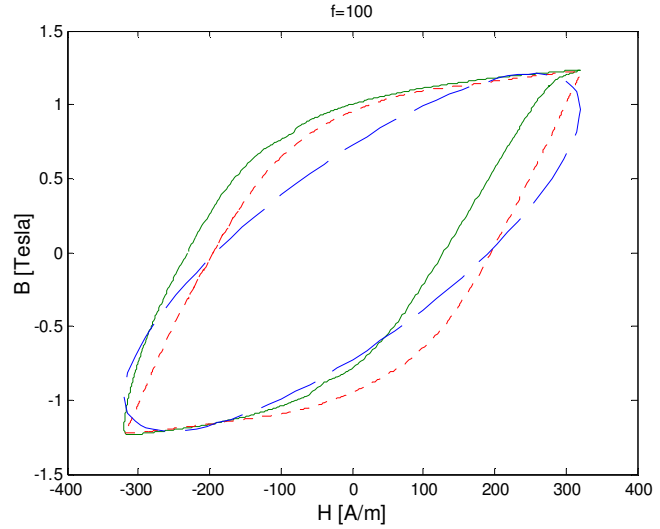
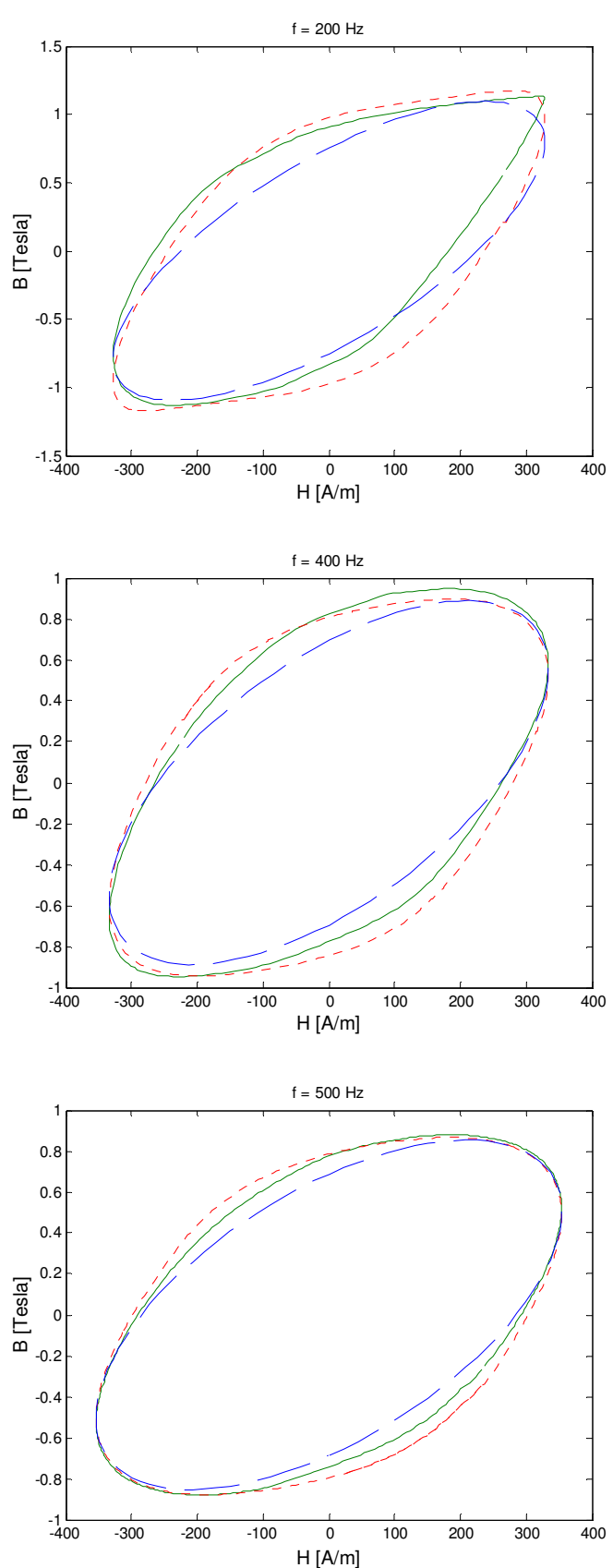

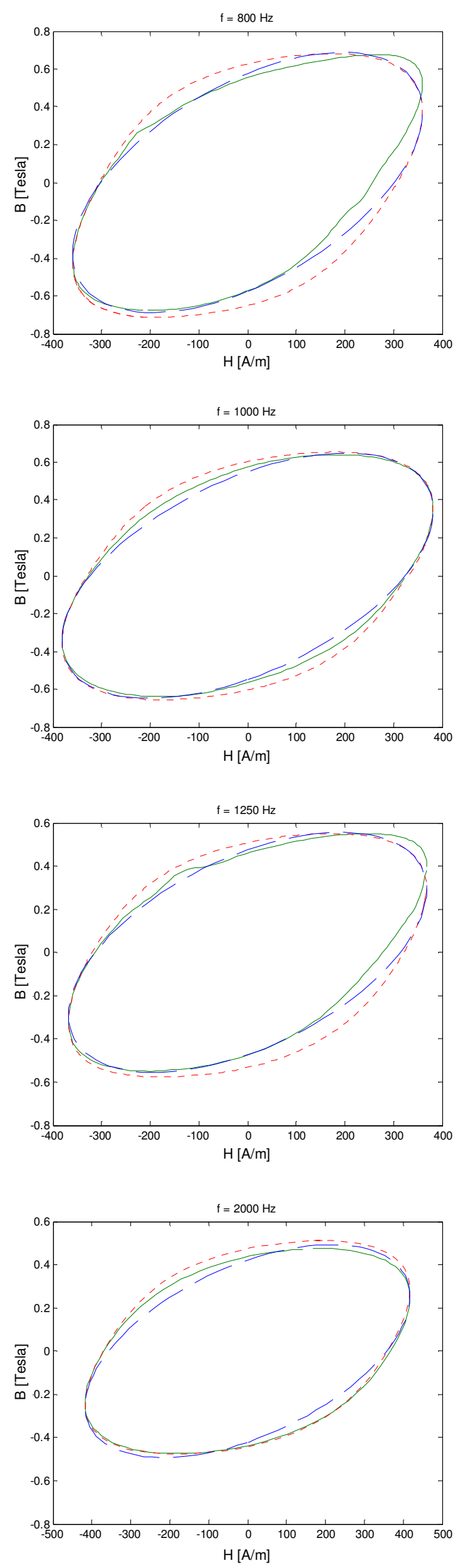

Fig. 7. $\quad H-B$ curves from measurements (solid green line), detailed model (dotted red line) and complex- $\mu$ model (dashed blue line) with $\mu$ calculated from expression (10), at different frequencies ranging from $50 \mathrm{~Hz}$ to $2 \mathrm{kHz}$.

The above way of defining a "best fit" of ellipses to the more complicated $H-B$ hysteresis relations approximately preserves both $H$ and $B$ amplitudes and magnetic losses in the whole frequency range. This is illustrated in the Figure 7, where the measured $H-B$ curves are compared with the corresponding complex- $\mu$ ellipses and the detailed model at different frequencies. As can be seen the simple model agrees very well with the measurements as long as saturation is not too strong, which means for low amplitude fields and/or for frequencies higher than about $200 \mathrm{~Hz}$.

\section{CONCLUSIONS}

In this paper, a simple, frequency dependent complex- $\mu$ model of magnetic core material has been developed and adjusted to measurements. Its real and imaginary parts were compared to measurements in a wide frequency range. The agreement was found satisfactory, especially for higher frequencies, which makes the complex- $\mu$ model a very convenient starting point for the estimation of flux distribution and losses in complicated core geometries.

Furthermore, $H-B$ curves from our measurements, the simple complex $\mu$ model and the detailed hysteresis model were compared for different frequencies. Again the results from the complex $\mu$ model were found to agree well with measurements at higher frequencies. At low frequencies and high field amplitudes the complex $-\mu$ model deviates from measurements and detailed hysteresis model, since it does not take saturation effects properly into account. This is, however, not expected to affect its usefulness for loss estimation.

\section{REFERENCES}

[1] G. Bertotti, "Hysteresis in Magnetism", Academic Press, San Diego, 1998.

[2] D. Ribbenfjärd , "A lumped transformer model including core losses and winding impedances", Licentiate Thesis in Electromagnetic Engineering, Stockholm, Sweden, 2007.

[3] D. Ribbenfjärd, G. Engdahl, "Novel Method for Modelling of Dynamic Hysteresis", IEEE Transactions on Magnetics, Vol. 44, No. 6, pp. 854857, June 2008.

[4] J. D. Jackson, "Classical Electrodynamics", third edition, John Wiley \& Sons, Inc., New York, 1998.

[5] R. L. Stoll, "The analysis of eddy currents", Clarendon press, Oxford, 1974.

[6] A. Bergqvist, "A phenomenological differential-relation-based vector hysteresis model", Journal of Applied Physics, Vol. 75, No. 10, pages 5484-5486, May 1994.

[7] A. Bergqvist, "Magnetic vector hysteresis model with dry friction-like pinning”, Physica B: Condensed Matter, Vol. 233, No. 4, pp. 342-347, 1997.

[8] E. Barbisio, F. Fiorillo, C. Ragusa, "Predicting Loss in Magnetic Steels Under Arbitrary Induction Waveform and With Minor Hysteresis Loops", IEEE Transactions on Magnetics, Vol. 40, No. 4, pp. 18101819 , July 2004

[9] F. de León, A. Semlyen, "Complete Transformer Model for Electromagnetic Transients", IEEE Transactions on Power Delivery, Vol. 9, No. 1 pp. 231-239, January 1994 